\title{
CORRELAÇÃO ENTRE ÍNDICE DE MASSA CORPORAL (IMC) E VELOCIDADE EM SALTOS ALTERNADOS EM CRIANÇAS
}

\author{
Danilo Cesar Machado ${ }^{1}$ \\ Jéssica Reis Buratti ${ }^{2}$ \\ Luiz Marcelo Ribeiro da $\mathrm{Luz}^{3}$ \\ Orcizo Francisco Silvestre ${ }^{4}$ \\ Alessandro de Freitas ${ }^{5}$ \\ Marcelo Braz Vieira ${ }^{6}$ \\ Paulo Ferreira de Araújo ${ }^{7}$ \\ José Irineu Gorla ${ }^{8}$
}

MACHADO, D. C.; BURATTI, J. R.; LUZ, L. M. R. da; SILVESTRE, O. F.; FREITAS, A. de; VIEIRA, M. B.; ARAÚJO, P. F. de; GORLA. J. I. Correlação entre índice de massa corporal (imc) e velocidade em saltos alternados em crianças. Arq. Cienc. Saúde UNIPAR, Umuarama, v. 23, n. 1, p, 3-8, jan./abr. 2019.

\begin{abstract}
RESUMO: A obesidade infantil vem em uma crescente pelo mundo inteiro e acredita-se que pode ser desecandeada pelo efeito negativo que os baixos níveis da coordenação motora podem causar sobre os níveis de atividade física e aptidão física durante a infância. Estudos analisaram a relação entre coordenação motora e composição corporal, e apontaram uma carência nas investigações sobre a influência do peso corporal em tarefas motoras específicas. Mediante ao exposto, o objetivo do presente estudo foi analisar a relação entre o Índice de Massa Corporal (IMC) e a velocidade em saltos alternados em crianças. Participaram do estudo 98 escolares com idade entre 6 e 10 anos. Para avaliação da velocidade de saltos alternados utilizou-se de uma das provas do teste Teste de Coordenação Corporal para Crianças (Köperkoordination Test für Kinder - KTK) que avalia saltos laterais por meio de teste específico. A análise estatística foi realizada por meio do programa R- Plus, o qual foi aplicado o teste de Mann-Whitney para verificar as diferenças do quociente motor (QM) entre meninos e meninas, Spearman para correlação entre o QM e IMC; e ANOVA para as diferenças entre a classificação do peso e o QM, sendo adotado nível de significância de $5 \%(p<0,05)$. Os resultados demonstraram não haver diferença estatística significativa entre os sexos para as variáveis analisadas. Entretanto, os meninos atingiram valores maiores para idade, IMC e QM. Na correlação entre o IMC e QM dos saltos laterais das crianças avaliadas, houve correlação negativa fraca, demonstrando que o IMC não teve grande influência no desempenho motor dos saltos quando analisado ambos os gêneros juntos. Tais resultados demonstram não haver correlação do IMC e a habilidade de saltos laterais em crianças com IMC elevado quando analisados de maneira geral. Porém, quando analisado por gênero, os meninos demonstram uma relação inversamente proporcional de IMC e saltos laterais o que não observou-se nas meninas.
\end{abstract}

PALAVRAS-CHAVE: Habilidade motora. Composição Corporal. Coordenação Motora.

\section{CORRELATION BETWEEN BODY MASS INDEX (BMI) AND SPEED IN ALTERNATED JUMPS IN CHILDREN}

ABSTRACT: Child obesity has been rising worldwide and is believed to be triggered by the negative effect that low levels of motor coordination can cause on the levels of physical activity and physical fitness during childhood. Studies analyzed the relationship between motor coordination and body composition, and pointed to a lack of research on the influence of body weight on specific motor tasks. The purpose of this study was to analyze the relationship between Body Mass Index (BMI) and the speed in alternate jumps in children. A total of 98 students aged 6 to 10 years took part in the study. In order to evaluate the speed in alternate jumps, one of the Köperkoordination Test für Kinder (KTK) tests that evaluates side jumps by means of a specific test was used. Statistical analysis was performed using the R-Plus program, which applied the Mann-Whitney test to verify the motor quotient (QM) differences between boys and girls, the Spearman test for correlation between QM and BMI; and ANOVA for the differences between weight classification and QM; as well as adopting a 5\% $(\mathrm{p}<0.05)$ significance level. The results showed that there were no significant statistical differences between the sexes for the analyzed variables; however, boys scored higher values for age, BMI and QM. A weak negative correlation was observed between BMI and QM for the lateral jumps of the assessed children, demonstrating that the BMI did not have a great influence on the motor performance of the jumps when both genders were analyzed together. These results show that there is no correlation between BMI and lateral jumping skills in children with high BMI when analyzed in general, but when analyzed by gender, boys demonstrate an inversely proportional relation of BMI and lateral jumps, which was not observed in girls.

KEYWORDS: Motor Skills. Body Composition. Motor Coordination.

\section{Introdução}

A obesidade na infância está aumentando rapidamente no mundo inteiro (LOPES et al., 2011), de acordo com Onis, Blossner, Borghi (2010), aproximadamente 43 milhões de crianças possuem sobrepeso ou obesidade e 92 milhões em risco de sobrepeso, com estimativa para 2020 de $60 \mathrm{mi}-$ lhões de crianças obesas e sobrepesadas.

A Organização Mundial de Saúde (OMS) em seu relatório intitulado Panorama da Segurança Alimentar e Nutricional na America Latina e Caribe (FAO/OPS, 2016) reafirma a estimativa do aumento da obesidade em crianças. O

DOI: 10.25110/arqsaude.v23i1.2019.6148

${ }^{1}$ Profissional de Educação Física. Rua Volta Redonda no 233, Colina do Sol, Catanduva - SP. Email: danilocmachado@gmail.com

${ }^{2}$ Profissional de Educação Física. Rua Itabirito, 1342, Jardim Ipiranga, Americana - SP. Email: danilocmachado@gmail.com

${ }^{3}$ Profissional de Educação Física. Rua Joviniano Barbosa, 35. Vila Hollandia, Campinas - SP. E-mail: luiz.daluz@globo.com

${ }^{4}$ Profissional de Educação Física. Rua Eucalipto, 472, Parque dos Pinheiros, Hortolândia - SP. E-mail: orcizocustodio@gmail.com

${ }^{5}$ Profissional de Educação Física. Rua Evangelina, 1261 apto 124, Vila Carrão, São Paulo - SP. E-mail: ale.educacaofisica@uninove.br

${ }^{6}$ Profissional de Educação Física. Av. João Paulo 710, 203A, torre 1, Florianópolis - SC. E-mail: marcelodego@yahoo.com.br

${ }^{7}$ Professor Dr. Universidade Estadual de Campinas. Avenida Érico Veríssimo, 701- Cidade Universitária, Campinas - SP. E-mail: paulof@fef.unicamp.br

${ }^{8}$ Professor Dr. Universidade Estadual de Campinas. Avenida Érico Veríssimo, 701- Cidade Universitária, Campinas - SP. E-mail: jigorla@uol.com.br 
documento com base nos dados da OMS indica aumento do sobrepeso infantil estimando que $7,3 \%$ das crianças menores de cinco anos estão acima do peso, sendo que destas a maior incidência está nas meninas com 7,7\%.

Em âmbito nacional, o Brasil apresenta dois quadros antagônicos: o da desnutrição e obesidade. Observa-se que, nos últimos cinquenta anos houve uma redução da desnutrição e aumento do sobrepeso e obesidade, mesmo em famílias de baixa renda. Em 1975, cerca de 8\% de crianças e adolescentes apresentavam desnutrição e 4\% obesidade, quadro que se inverteu nos últimos anos, apontando $9 \%$ com obesidade e 3\% desnutrição (BARBOSA, 2009). De acordo com o Instituto Brasileiro de Geografia e Estatística (IBGE), em 2009, cerca de um terço das crianças brasileiras de 5 a 9 anos apresentavam excesso de peso corporal (33,5\% sobrepesadas e $14,3 \%$ obesas) e $20,5 \%$ dos adolescentes com idade entre 10 a 19 anos estavam com sobrepeso e $4,9 \%$ obesos (BRASIL, 2010).

As relações diretas entre ausência de atividade fisica e obesidade se mostram importantes para compreender os mecanismos ligados aos fatores que podem influenciar o nível de atividade e determinar as causas que levam à inatividade física. À medida que surgem ao longo do tempo de desenvolvimento um caminho potencial causal que tem sido praticamente ignorado na literatura, o fato da influência do desenvolvimento de habilidades motoras em níveis de atividade física (STODDEN; GOODWAY, 2007, D'HONDT et al., 2013), acredita-se na importância de compreender tais fatores e a influência dessas variáveis.

Partindo do pressuposto que a ausência de atividade física pode conduzir a um menor desempenho físico e motor, e ainda a um declínio no rendimento físico, podendo assim produzir frustração e fuga das aulas de educação física ou atividade de lazer, é possível que crianças com dificuldades no desempenho de habilidades motoras tenham menores chances de participação em atividades motoras escolares, diminuindo seu engajamento motor e a participação futura em atividades físicas que asseguram à qualidade de vida e, consequentemente a manutenção do peso corporal (POETA et al., 2010, MELLO; LOPES, 2013).

A obesidade desta forma pode ser desecandeada pelo efeito negativo que os baixos níveis de coordenação motora causa sobre os níveis de atividade física, aptidão física e percepção de competência física durante a infância, resultando em baixo sucesso motor, levando a um estilo de vida inativo e, consequentemente ao sedentarismo e obesidade (STODDEN et al., 2008).

Estudos (CATENASSI et al., 2007, BUSTAMANTE et al., 2008, MARTINS et al., 2010, LOPES et al., 2011, D'HONDT et al., 2014) analisaram a relação entre coordenação motora e composição corporal, entretanto, observa-se uma carência de investigações sobre a influência do peso corporal em tarefas motoras específicas. Diante disso, o objetivo do presente estudo foi verificar a relação entre a classificação do peso corporal e a velocidade em saltos alternados em crinaças, por meio de uma das provas do Teste de Coordenação Corporal para Crianças (Körperkoordination Test für Kinder - KTK), específico de saltos laterais.

\section{Metodologia}

A amostra foi composta por 98 crianças (49 meninos e 49 meninas) de 6 a 10 anos de idade, matriculados em uma escola pública de ensino regular da cidade de Campinas - SP. O presente estudo foi aprovado pelo Comitê de Ética da Universidade Estadual de Campinas, registrado com protocolo n.272/2011, e um termo de consentimento livre e esclarecido assinado pelos pais ou responsáveis legais, autorizando a participação.

Para o cálculo do IMC, foram mensuradas as medidas da estatura e peso utilizando estadiômetro e balança dentro dos padrões de regularidade, com precisão de $0,1 \mathrm{~cm}$ e 100 gramas, respectivamente. O IMC foi classificado de acordo com o protocolo proposto por Cole et al. (2000).

A velocidade em saltos alternados foi avaliada por meio de uma das provas Teste de Coordenação Corporal para Crianças (Körperkoordination Test für Kinder - KTK), que consiste em saltar de um lado para o outro da plataforma com os dois pés ao mesmo tempo, o mais rápido possível, durante 15 segundos, sobre uma plataforma de madeira de $60 \times 50 \times 0$, oito $\mathrm{cm}$ e um sarrafo de $60 \times 4 \times 2 \mathrm{~cm}$, dividindo a plataforma em duas partes iguais. Cada criança realizou um ensaio com cinco saltos e teve duas tentativas válidas para execução, sendo contabilizada a quantidade de saltos realizados pelos sujeitos, para posteriormente classificar o quociente motor relacionado à idade cronológica.

Para o tratamento estatístico foi utilizado o programa R Plus versão 2.14.0. Os dados foram submetidos ao teste de normalidade de Komolgorov-Smirnov e apresentados pela mediana e amplitude interquartil. As diferenças do QM entre meninos e meninas foram analisadas por meio do teste de Mann-Whitney; e a correlação entre o QM e IMC através do teste de Spearman. Para as diferenças entre a classificação do peso e o QM foi utilizado o teste ANOVA. Nível de significância adotado de $5 \%(p<0,05)$

\section{Resultados}

Na tabela 1 são apresentados os valores médios de idade, IMC e QM da amostra estudada. Através dos valores de $p$ obtidos pelo teste de Mann Whitney, nota-se que não houve diferença estatística significativa entre os sexos para as variáveis analisadas. Entretanto, meninos atingiram valores maiores para idade, IMC e QM.

Tabela 1: Características da amostra estudada, valores médios, desvio padrão e teste de Mann Whitney para identificar possíveis diferenças entre sexo.

\begin{tabular}{lccc}
\hline & Meninos & Meninas & P \\
\hline Idade & $7,76 \pm 1,0$ & $7,69 \pm 1,2$ & 0,96 \\
IMC & $17,49 \pm 4,6$ & $16,75 \pm 3,2$ & 0,27 \\
QM & $89,53 \pm 15,6$ & $81,35 \pm 21,9$ & 0,09 \\
\hline
\end{tabular}

Em relação à classificação da composição corporal, a figura 1 demonstra a prevalência de peso normal, sobrepesados e obesos na amostra de acordo com o protocolo proposto por Cole et al., (2000). Observou-se que $73 \%$ dos avaliados foram classificados com peso normal, 16\% com sobrepeso e $11 \%$ obeso, sendo que os meninos atingiram 
maiores valores de IMC (21\% com sobrepeso e $12 \%$ obesos) do que as meninas (12\% com sobrepeso e $10 \%$ obesas).

Figura 1: Percentual da amostra de acordo com a classificação do peso.

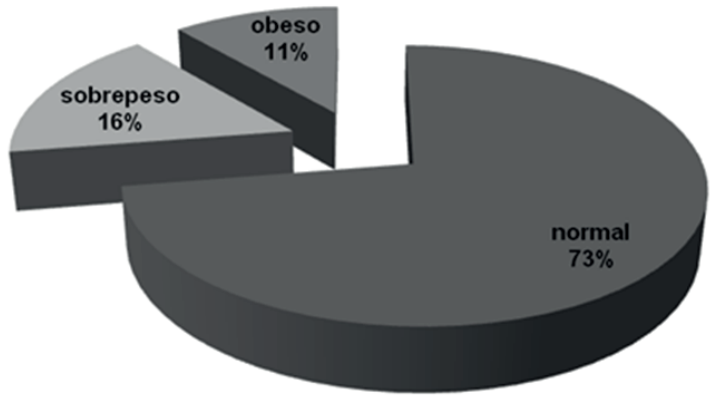

A Tabela 2 apresenta a correlação entre o IMC e QM dos saltos laterais das crianças avaliadas. Nota-se correlação negativa fraca $(\mathrm{r}=-0.076)$ sem estatística significativa $(\mathrm{p}=$ $0,46)$, demonstrando que o IMC não teve grande influência no desempenho motor dos saltos laterais.

Quando realizada a correlação entre as variáveis separadas por gênero, observa-se comportamento distinto. Para os meninos, o coeficiente de correlação foi negativo $(\mathrm{r}=-0,285)$ e fraco, ainda que estatisticamente significativo $(p<0,05)$. Já para o sexo feminino, foi encontrada relação fraca $(\mathrm{r}=0,064)$ e positiva, sem significância estatística $(\mathrm{p}=0,66)$, demonstrando uma tendência de influência negativa do IMC sobre a tarefa motora nos meninos e praticamente nula nas meninas.

Tabela 2: Valores de coeficiente de correlação de Spearman entre IMC e QM de saltos laterais da amostra avaliada, de meninos e meninas.

\begin{tabular}{ccccccc}
\hline & $\begin{array}{c}\text { Meninos e } \\
\text { Meninas } \\
(\mathbf{n = 9 8 )}\end{array}$ & \multicolumn{2}{c}{$\begin{array}{c}\text { Meninos } \\
(\mathbf{n = 4 9 )}\end{array}$} & \multicolumn{2}{c}{$\begin{array}{c}\text { Meninas } \\
(\mathbf{n}=49)\end{array}$} \\
\hline $\mathrm{QM}$ & $\mathrm{R}$ & $\mathrm{p}$ & $\mathrm{R}$ & $\mathrm{p}$ & $\mathrm{R}$ & $\mathrm{p}$ \\
& $-0,076$ & 0,46 & $-0,285$ & 0,05 & 0,064 & 0,66 \\
\hline
\end{tabular}

\section{Discussão}

Os dados de sobrepeso e obesidade encontrados no presente estudo, comparados ao estudo realizado por Pellegrini et. al. (2010), que verificaram o sobrepeso e a obesidade em escolares de sete a nove anos das cinco regiões brasileiras, são próximos ao da média nacional na faixa de sobrepeso, sendo de $16 \%$ e $15,4 \%$ respectivamente, e na obesidade os dados foram de $11 \%$ e $7,8 \%$ respectivamente.

Tais resultados quando comparados à Região $\mathrm{Su}-$ deste mostraram-se relativamente piores, como pode ser observado no estudo de Pelegrini et. al. (2006) em que 10,2 \% das crianças apresentaram sobrepeso e $2,9 \%$ obesidade. Em contrapartida, os dados do presente estudo foram menores que os valores brasileiros do POF 2008-2009, mas dev-se ter cautela, já que Tassitano, Tenório, Hallal (2009) verificaram que os maiores números de adolescentes obesos estão na região sul e sudeste do país.
Um fato que não foi considerado no presente estudo, mas que pode ser um fator importante a ser considerado sejam os fatores internos e externos, que Barbosa (2009) em seu estudo cita que fatores internos (genéticos e metabólicos) ou externos (alimentares, emocionais ou psicológicos e os níveis de atividade física) são fatores que isoladamente ou que na maior parte das vezes combinados resultam na obesidade. Dessa forma, podendo ser um fator que esteja corroborando com o aumento da obesidade e sobrepeso.

Os valores encontrados no presente estudo da prova saltos laterais apresentados na estatística descritiva vão ao encontro dos verificados por estudos de Bustamante (2008) e Vandorpe (2010). Nesses estudos também observou-se favorecimento dos meninos em relação às meninas, mas sem diferenças significativas. Já no estudo realizado por Lopes et. al. (2003) que analisaram o nível de desenvolvimento da coordenação motora de crianças de 6 a 10 anos, na prova saltos laterais, não houve diferenças significativas entre meninos e meninas.

$\mathrm{Na}$ análise da relação entre o IMC e a agilidade em saltos alternados, os resultados demonstram que quando não se fez diferença de gêneros, observou-se relação negativa, fraca e sem diferença significativa. Dados muito próximos foram verificados por Catenassi et al. (2007) que encontraram correlação fraca e sem diferença significativa entre o IMC e a agilidade em saltos alternados sem distinção de gênero. Bustamente et al. (2008) verificaram em um de seus estudos que em todas as quatro provas do KTK a adiposidade influenciou negativamente na pontuação, mas na prova de SL a diferença encontrada foi pequena.

Quando analisada a relação das variáveis QM e IMC com os gêneros separados, os meninos apresentaram correlação negativa estatisticamente significativa, mas fraca, demonstrando que o estado nutricional não teve grande influência na coordenação motora. Lopes et al., (2011) corroboram com estes achados ao avaliar a relação entre IMC e coordenação motora de 3.616 meninos portugueses de 6 a 14 anos de idade utilizando-se do teste de KTK para mensurar o desempenho motor, que demonstrou correlação fraca, negativa e estatisticamente significativa com o IMC $(\mathrm{r}=<$ 0,05 a 0,49 ). Os autores também observaram que as crianças e adolescentes com peso normal atingiram maior valor da coordenação motora quando comparado ao grupo com sobrepeso e obesidade.

Na Alemanha, Graf et al., (2003) analisaram a relação entre o IMC e coordenação motora por meio do teste KTK de 341 meninos com idade média de 6,75 $\pm 0,43$, o qual observou que os valores de coordenação motora no grupo de sobrepeso e obeso foram menores quando comparado ao grupo com peso normal, sendo observada relação negativa significativa entre IMC e QM $(r=<0,165)$.

D'Hondt et al. (2011) realizaram um estudo longitudinal com o objetivo de verificar a relação entre peso corporal e coordenação motora. Para tanto, os autores avaliaram 24 meninos e 12 meninas belgas com idade média de 10,5 $\pm 1,4$ anos. Os avaliados foram submetidos a um programa de prevenção da obesidade durante 6 a 10 meses, incluindo restrição alimentar, apoio psicológico e atividade física regular. Para a avaliação da coordenação motora foi utilizado o teste KTK e a classificação do peso corporal foi realizada pelo IMC. No pré-teste, foi observada diferença estatística entre 
o grupo com peso corporal normal, sobrepesados e obesos, demonstrando relação inversa entre IMC e coordenação motora. Entretanto, após o período de intervenção, os autores relatam ganhos na coordenação motora de aproximadamente $27 \%$ no grupo de crianças com sobrepeso e obesas, devido à redução dos valores de IMC.

Em um estudo somente com meninos, Vandendriessche et al. (2011). Analisaram a influência de características morfológicas e de aptidão física nos níveis de coordenação motora de 613 sujeitos de 7 a 11 anos. A massa corporal foi mensurada por meio da bioimpedância elétrica e a aptidão física consistiu na realização de diversos testes da bateria EUROFIT (preensão manual, sentar e alcançar, salto horizontal, shuttle run e corrida de 20 metros). Para mensurar a coordenação motora, foi adotado o teste KTK. Os autores observaram que o percentual de gordura demonstrou relação inversa nas quatro tarefas compreendidas pelo KTK e relação direta com aptidão física. Com isso, os autores concluíram que maior nível de percentual de gordura resulta em menor nível de coordenação motora. Entretanto, quanto maior os valores de aptidão física, melhor a coordenação motora.

Ao contrário dos meninos, as meninas apresentaram uma fraca correlação positiva e estatisticamente não significativa, estes dados estão de acordo com os verificados por Machado; Campos, Silva (2002), que analisaram a relação da composição corporal por meio de pregas cutâneas e a habilidade de locomoção e manipulação. Para tanto, foram avaliadas 80 crianças do sexo feminino de 5 a 8 anos matriculadas na rede de ensino de Curitiba - Paraná, Brasil. Foi observado que a gordura corporal não influenciou estatisticamente os valores atingidos nos testes de locomoção e manipulação das meninas, atingindo valores de $\mathrm{r}$ variando de 0,05 a 0,10 .

Em outro estudo brasileiro, Catenassi et al. (2007), verificaram a relação entre o desempenho em tarefas de habilidade motora grossa com o IMC, de 11 meninas com ida-

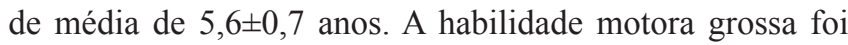
mensurada através do Testo of Gross Motor Development TGMD (ULRICH, 2000) e o teste de KTK. Os autores não encontraram correlações significativas entre IMC e os valores atingidos pelo TGMD e KTK, alcançando valores de $r$ variando de 0,43 a 0,42 , indicando um potencial igualitário de desenvolvimento motor para todas as crianças, não sendo restringido pela adiposidade corporal.

Neste sentido, nota-se uma discordância entre estudos no que se refere à influência da adiposidade corporal na coordenação motora de crianças e adolescentes. Dentre os fatores que possam gerar esta discrepância, destacam-se as amostras reduzidas de algumas investigações, apresentando baixa prevalência de obesidade e sobrepeso. Outro fator que dificulta a interpretação dos dados entre a composição corporal e coordenação motora é o método adotado para mensurar o estado nutricional das crianças e adolescentes.

O IMC é um dos métodos mais usualmente utilizados e é considerado atualmente um bom indicador para diagnosticar o sobrepeso e a obesidade. Pode-se dizer que o seu frequente uso pode se dar pelo fato de apresentar baixo custo operacional; ser de fácil aplicação, e sendo assim facilmente realizado no ambiente escolar, sendo de fácil acesso ao professor de Educação Física; não ser invasivo e ainda ter possibilidade de comparações com tabelas de referências. Contudo, deve-se ter cautela ao analisar os resultados, uma vez que o índice pode apresentar um potencial discriminatório relativamente limitado para a identificação dos casos de obesidade (DIETZ; BELLIZZI, 1999; CATENASSI et al., 2007).

Januário et al. (2008) compararam o IMC e as dobras cutâneas como indicadores de obesidade em escolares de 8 a 10 anos e concluíram que há concordância moderada para classificar crianças em relação à obesidade. Em contrapartida, Glaner (2005) concluiu que o IMC não apresenta resultados consistentes para classificar a gordura corporal. Guedes; Rechenchosky (2008) realizaram um estudo que analisou a relação do IMC e a espessura de dobras cutâneas como preditores da gordura corporal, as quais verificaram que apesar dos valores expressos por ambos serem similares quando analisado grupos, individualmente pode apresentar valores diferentes, concluindo a necessidade de prudência ao interpretar os dados.

Em um estudo português transversal que avaliou 794 crianças de seis a nove anos de ambos o sexo, Melo e Lopes (2013) verificaram que as crianças com sobrepeso e obesidade apresentaram baixos níveis de coordenação comparadas às com peso normal, havendo uma correlação negativa entre IMC e coordenação motora, com um aumento da correlação ocorrendo entre os seis e os oito anos de idade em ambos os sexos e; entre os seis e os nove anos nas meninas, sendo as correlações de baixa a moderada, concluindo que a coordenação está negativamente correlacionada ao IMC e que esta aumenta durante a infância.

Em uma pesquisa com 3344 meninos e 3281 meninas portuguesas realizada por Lopes, Stodden, Rodrigues (2014), analisaram-se as associações transversais entre coordenação motora e o status do peso através do IMC, observaram-se que crianças com níveis baixos de coordenação motora demonstraram um maior risco de sobrepeso ou obesidade, em comparação com crianças com coordenação motora alta, e que o risco aumentaria em toda a faixa etária de idades em ambos os sexos. Em conclusão demonstrou a necessidade de estratégias para intervir em crianças que apresentam coordenação motora baixa em uma idade precoce.

Luz et al. (2015), realizou uma revisão sistemática com meta-análise que teve como objetivo verificar a relação entre IMC e a coordenação motora por meio do KTK em crianças e jovens escolares saudáveis, de estudos em português e inglês indexados nas bases de dados eletrônicas PubMed e Scielo. No total foram 10 estudos incluídos concluindo que associação entre o IMC e o KTK foi classificada como pequena para o sexo masculino e moderada para o sexo feminino, e que há uma tendência positiva entre maiores valores de IMC e menores resultados da coordenação motora.

Em novo estudo de Luz et al. (2015), feito com 73 crianças do sexo masculino e 8 anos de idade que teve como objetivo analisar a associação do estado maturacional com o desempenho nas provas do KTK. Os resultados indicaram que estatura, massa corporal, perímetro de cintura e massa gorda apresentaram correlação inversa de magnitude pequena a moderada com a provas do KTK, sendo a relação do IMC com os saltos laterais significativa $(\mathrm{r}=-0,30)$.

Freitas et al. (2017) em um estudo analisaram a relação entre o IMC e a coordenação motora de jovens atletas de atletismo levando em conta a maturação somática, observou que não houve relação entre a coordenação motora e a matu- 
ração somática, mas por outro lado obteve correlação negativa e estatisticamente significativa entre o IMC e a coordenação motora, indicando que com o aumento do IMC há uma diminuição da coordenação motora, contudo ao inspecionar o gráfico de dispersão entre o IMC e a coordenação motora, sugeriu que essa correlação é significativa para valores de IMC acima de 20,6; concluindo que o excesso de peso prejudica a coordenação motora e que o treinamento por si só não é suficiente para que jovens, com excesso de peso, tenham o mesmo desempenho motor de jovens com peso normal.

Em um estudo dinamarquês de Lima et al. (2017), que teve como objetivo analisar a estabilidade da coordenação motora em crianças de idade entre 6 a 13 anos de idade por meio de avaliações regulares, evidenciou que a chance de crianças com IMC mais alto foi 5,44 vezes maior de estar no grupo de classificação coordenação mais baixo durante o período de 7 anos em comparação com crianças com IMC mais baixo; concluindo que as crianças mais pesadas tiveram maior chance de estar no grupo de coordenação motora menor nas idades posteriores e que a intervenção pode ser útil em idades anteriores para aqueles com coordenação menor e status de peso desvantajoso.

Em outro estudo de Lima et al. (2017), cujo objetivo foi investigar a associação longitudinal entre a atividade física, competência motora, aptidão cardiorrespiratória e gordura corporal de crianças com idade entre 6 e 13 anos, revelou que a competência motora e a aptidão cardiorrespiratória influenciaram diretamente o desenvolvimento da gordura corporal, concluindo que uma vez que atividade física, competência motora e aptidão cardiorrespiratória estiveram associadas longitudinalmente com gordura corporal, indicando que é necessário que ocorram intervenções com atividades físicas apropriadas que promovam o desenvolvimento da aptidão física e da competência motora.

\section{Coniderações Finais}

As crianças com Índice de Massa Corporais elevados participantes do estudo, de modo geral não apresentaram correlação do IMC e a habilidade de saltos laterais quando analisado em grupo, já quando se observou por gênero, os meninos demonstram uma relação inversamente proporcional de IMC e os Saltos Laterais, já as meninas não apresentaram relação ao comparado com crianças sem sobrepeso ou obesidade.

A habilidade motora avaliada não mostrou ter relação com o índice de Massa Corporal quando considerado a população total. Porém, acredita-se que tal fato sugere que novas pesquisas envolvendo um número consideravelmente maior de crianças sejam necessárias, a fim de apontar a existência dessa relação, como sugere outros estudos, e ainda dessa forma conscientizar a importância de uma vida ativa e saudável, detectando as possíveis interferências no desenvolvimento motor de crianças com sobrepeso e obesas.

As limitações deste estudo estão relacionadas com o número de participantes, e o fato de se utilizar o IMC como parâmetro para identificar as crianças sobrepesadas e obesas, visto a fragilidade do método. Contudo, acredita-se que novos estudos avaliando outras variáveis motoras como equilíbrio, coordenação motora global seriam de grande valor para identificar possíveis déficits motores.

\section{Referências}

BARBOSA, V. L. P. Prevenção da obesidade na infância e na adolescência: exercícios, nutrição e psicologia. 2. ed. Barueri, SP: Manole, 2009.

BRASIL. Ministério do Planejamento, Orçamento e Gestão, Instituto Brasileiro de Geografia e Estatística, Pesquisa de orçamentos familiares 2008-2009. Antropometria e Estado Nutricional de Crianças, adolescentes e adultos no Brasil. Rio de Janeiro: IBGE; 2010.

BUSTAMANTE, A. V.; et al. Coordinación motora: influencia de la edad, sexo, estatus socio-económico y niveles de adiposidad en niños peruanos. Revista Brasileira de Cineantropometria \& Desempenho Humano, [s.l], p. 25-34, 2008.

CATENASSI, F. Z. et al. Relação entre índice de massa corporal e habilidade motora grossa em crianças de quatro a seis anos. Rev Bras Med Esporte, v. 13, n.4, Jul/Ago, 2007.

COLE, T. J. et al. Establishing a standard definition for child overweight and obesity worldwide: international survey. BMJ, [s.1], v. 320, p. 1-6, 2000.

DIETZ, W. H.; BELLIZZI, M. C. Introduction: the use of body mass index to assess obesity in children. Am J Clin Nutr., [s.1], v.70 (suppl), p. 123S-5S, 1999.

D'HONDT, E. et al. Weight Loss and Improved Gross Motor Coordination in Children as a Result of Multidisciplinary Residential Obesity Treatment. Obesity, [s.1.], v. 19, n. 10, p.1999-2005, 30 jun. 2011.

D'HONDT, E. et al. A longitudinal analysis of gross motor coordination in overweight and obese children versus nomalweight perrs. International Journal of Obesity, [s.1], v.37, p.61-67, 2013.

D'HONDT, E. et al. A longitudinal study of gross motor coordination and weight status in children. Obesity,[s.l],v. 22, n.06, p.1506-1511, 2014.

FAO/OPS. América Latina y el Caribe - Panorama de la seguridade alimentaria y nutricional: Sistemas alimentarios sostenibles para poner fin al hambre y la malnutrición 2016. Disponível em: <http://www.fao.org/3/a-i6747s>.pdf Acesso em: $24 / 10 / 2017$

FREITAS, J. V. D. et al. Relação entre o excesso de peso e a coordenação motora de jovens atletas de atletismo. Rev. Bras Ciênc Esporte, [s.1], v.39, n.1, p. 91-97, 2017.

GLANER, M. F. Índice de massa corporal como indicativo da gordura corporal comparado às dobras cutâneas. Revista Brasileira de Medicina do Esporte, [s.1], v.11, n.4, 2005.

GRAF, C. et al. Correlation between BMI, leisure habits and motor abilities in childhood (CHILT-Project). International Journal Of Obesity, [s.1.], v. 28, n. 1, p.22-26, 2 dez. 2003 
GUEDES, D. P.; RECHENCHOSKY, L. Comparação da gordura corporal predita por métodos antropométricos: índice de massa corporal e espessuras de dobras cutâneas. Revista Brasileira de Cineantropometria \& Desempenho Humano, [s.1], v.10, n.1, p.1-7, 2008.

JANUÁRIO, R. S. B. et al. Índice de massa corporal e dobras cutâneas como indicadores de obesidade em escolares de 8 a 10 anos. Revista Brasileira de Cineantropometria \& Desempenho Humano, [s.1], v.10, n.3, p.266-270, 2008.

KIPHARD, E. J.; SCHILLING, V. F. The Body Coordination Test (BCT). Journal of Physical Education and Recreation, [s.1], p. 37, 1976.

LIMA, R. A. et al. Tracking of Gross Motor Coordination From Childhood Into Adolescence. Research Quarterly For Exercise And Sport, [s.1.], v. 88, n. 1, p. 52-59, 2 jan. 2017.

LIMA, R. A. et al. Motor competence and cardiorespiratory fitness have greater influence on body fatness than physical activity across time. Scandinavian Journal Of Medicine \& Science In Sports, [s.1.], p. 1-22, mar. 2017.

LOPES, V. P. et al. Estudo do nível de desenvolvimento da coordenação motora da população escolar (6 a 10 anos de idade) da Região Autónomados Açores. Revista Portuguesa de Ciências do Desporto, [s.1] v. 3, n. 1, p. 47-60, 2003.

LOPES, V. P. et al. Correlation between BMI and motor coordination in children. Journal of Science and Medicine in Sport, [s.1],v.15, n.1, p.38-4, 2011.

LOPES, V. P.; STODDEN, D. F.; RODRIGUES, L. P. Weight status is associated with cross-sectional trajectories of motor co-ordination across childhood. Child Care Health Dev., [s.1], v. 40, n. 6, p. 891-899-Nov, 2014.

LUZ, L. G. d. et al. Associação entre IMC e teste de coordenação corporal para crianças (KTK). Uma metaanálise. Revista Brasileira de Medicina do Esporte, [s.1], v. 21, n. 3, Mai/Jun, 2015.

MACHADO, H. D.; CAMPOS, W.; SILVA. G. Relação entre composição corporal e a performance de padrões motores fundamentais em escolares. Rev. Bras. Ativ. Fís. Saúde, [s.1], v. 7, n. 1, p. 63-70, 2002.

MARTINS, D. et al. Correlates of changes in BMI of children from the Azores islands. International Journal of Obesity, [s.1], v. 34, p. 1487-1493, 2010.

MELO, M. M.; LOPES, V. P. Associação entre o índice de massa corporal e a coordenação motora em crianças. Rev. Bras. Educ. Fís. Esporte, [s.1], v. 27, n. 1, p. 7-13, jan-mar, 2013.

ONIS, M.; BLOSSNER, M.; BORGHI, E. Global prevalence and trends of overweight and obesity among preschool children. American Journal of Clinical Nutrition, [s.1], v.
92, p. $1257-64,2010$.

PELLEGRINI, A. et al. Sobrepeso e obesidade em escolares brasileiros de sete a nove anos: dados do projeto Esporte Brasil. Revista Paulista de Pediatria, [s.1], v. 28, n. 3, p. 290-95, 2010.

POETA, L. S. et al. Desenvolvimento motor de crianças obesas. R. bras. Ci. e Mov; [s.1], v. 18, n. 4, p. 18-25, 2010.

STODDEN, D. F. et al. A Developmental Perspective on the Role of Motor Skill Competence in Physical Activity: An Emergent Relationship. Quest, [s.1], v. 60, p. 290-306, 2008.

STODDEN, D. F.; GOODWAY, J. D. The dynamic association between motor skill development and physical activity: poor motor-skill development, which limits success, may discourage physical activity.(Combating Obesity in K-12 Learners). The Journal of Physical Education, Recreation \& Dance, [s.1], v. 78, n. 8, p. 33-49, Oct, 2007.

TASSITANO, R. M.; TENÓRIO, M. C. M.; HALLAL, P. C. Revisão sistemática sobre obesidade em adolescentes brasileiros. Revista Brasileira de Cineantropometria \& Desempenho Humano, [s.1], p. 449-456, 2009.

ULRICH, D. A. Test of gross motor development. 2. ed. Austin: Pro-Ed; 2000.

VANDENDRIESSCHE, J. B. et al. Multivariate association among morphology, fitness, and motor coordination characteristics in boys age 7 to 11. Pediatr Exerc Sci., [s.1], v. 23, n. 4, p. 504-520-Nov, 2011.

VANDORPE, B. et al. The KörperkoordinationsTest für Kinder: reference values and suitability for 6-12-year-old children in Flanders. Scandinavian Journal of Medicine \& Science in Sports, [s.1], v. 21, p. 378-88, 2010.

Recebido em: 04/05/2017 Aceito em: 10/09/2018 